\title{
UMBRELLA protocol: systematic reviews of multivariable biomarker prognostic models developed to predict clinical outcomes in patients with heart failure
}

Maria D. L. A. Vazquez-Montes', Thomas P. A. Debray ${ }^{2,3}$, Kathryn S. Taylor', Benjamin Speich ${ }^{2,4}$, Nicholas Jones ${ }^{1}$, Gary S. Collins ${ }^{2,5}$, F. D. R. Richard Hobbs ${ }^{1}$, Emmanuella Magriplis ${ }^{2,6}$, Hugo Maruri-Aguilar ${ }^{7}$, Karel G. M. Moons ${ }^{2,3}$, John Parissis ${ }^{8}$, Rafael Perera ${ }^{1}$, Nia Roberts ${ }^{9}$, Clare J. Taylor ${ }^{1}$, Nikolaos P. E. Kadoglou ${ }^{2 \dagger}$, Marialena Trivella ${ }^{2 * \dagger}$ (D) and on behalf of the proBHF group

\begin{abstract}
Background: Heart failure (HF) is a chronic and common condition with a rising prevalence, especially in the elderly. Morbidity and mortality rates in people with HF are similar to those with common forms of cancer. Clinical guidelines highlight the need for more detailed prognostic information to optimise treatment and care planning for people with HF. Besides proven prognostic biomarkers and numerous newly developed prognostic models for HF clinical outcomes, no risk stratification models have been adequately established. Through a number of linked systematic reviews, we aim to assess the quality of the existing models with biomarkers in HF and summarise the evidence they present.
\end{abstract}

Methods: We will search MEDLINE, EMBASE, Web of Science Core Collection, and the prognostic studies database maintained by the Cochrane Prognosis Methods Group combining sensitive published search filters, with no language restriction, from 1990 onwards. Independent pairs of reviewers will screen and extract data. Eligible studies will be those developing, validating, or updating any prognostic model with biomarkers for clinical outcomes in adults with any type of HF. Data will be extracted using a piloted form that combines published good practice guidelines for critical appraisal, data extraction, and risk of bias assessment of prediction modelling studies. Missing information on predictive performance measures will be sought by contacting authors or estimated from available information when possible. If sufficient high quality and homogeneous data are available, we will metaanalyse the predictive performance of identified models. Sources of between-study heterogeneity will be explored through meta-regression using pre-defined study-level covariates. Results will be reported narratively if study quality is deemed to be low or if the between-study heterogeneity is high. Sensitivity analyses for risk of bias impact will be performed.

(Continued on next page)

\footnotetext{
* Correspondence: marialena.trivella@csm.ox.ac.uk

${ }^{\dagger}$ Nikolaos P. E. Kadoglou and Marialena Trivella contributed equally to this

work.

${ }^{2}$ Centre for Statistics in Medicine, Nuffield Department of Orthopaedics,

Rheumatology and Musculoskeletal Sciences, Botnar Research Centre,

University of Oxford, Windmill Road, Oxford OX3 7LD, UK

Full list of author information is available at the end of the article
}

(c) The Author(s). 2020 Open Access This article is licensed under a Creative Commons Attribution 4.0 International License, which permits use, sharing, adaptation, distribution and reproduction in any medium or format, as long as you give appropriate credit to the original author(s) and the source, provide a link to the Creative Commons licence, and indicate if changes were made. The images or other third party material in this article are included in the article's Creative Commons licence, unless indicated otherwise in a credit line to the material. If material is not included in the article's Creative Commons licence and your intended use is not permitted by statutory regulation or exceeds the permitted use, you will need to obtain permission directly from the copyright holder. To view a copy of this licence, visit http://creativecommons.org/licenses/by/4.0/. 
(Continued from previous page)

Discussion: This project aims to appraise and summarise the methodological conduct and predictive performance of existing clinically homogeneous HF prognostic models in separate systematic reviews. Registration: PROSPERO registration number CRD42019086990

Keywords: Acute heart failure, Decompensated heart failure, Chronic heart failure, Biomarkers, Prediction rule, Risk score, Prediction accuracy, CHARMS, PROBAST, Prognosis

\section{Background}

This in an umbrella protocol covering a number of systematic reviews in the area of heart failure. Clinically homogenous data will be considered together in each systematic review, while the clinical outcomes listed below will be explored where possible in all the resulting reviews.

\section{Heart failure epidemiology}

Heart failure (HF) is a complex disease related to a structural and/or functional cardiac abnormality which impairs the ability of the heart to function as an efficient blood pump. With a rising prevalence (currently estimated between 6 and 10\% in people older than 65 years) primarily due to population ageing, HF is now a major public health problem affecting approximately 26 million people worldwide [1-3]. In 2012, it was estimated that HF is responsible for health expenditures as high as 31 billion US\$ worldwide and costs seem to be rising [4].

People with HF may be categorised in terms of symptom stability. Acute HF (AHF) refers to either onset of symptoms in people with previously unknown HF (de novo HF) or to a recent decompensation of previously stable HF symptoms, in contrast to people with chronic HF (CHF) who have had an extended period of symptom stability. CHF may also be categorised according to the individual's left ventricular ejection fraction (LVEF) into: preserved ejection fraction (HFpEF) if $\mathrm{LVEF} \geq 50 \%$, midrange ejection fraction (HFmrEF) if LVEF ranges between 40 and $49 \%$, and reduced ejection fraction (HFrEF) if LVEF $<40 \%$ [5].

People with HF may require hospitalisations and frequent re-admissions [6]. In the United Kingdom, CHF accounts for $2 \%$ of all National Health Service (NHS) hospital admissions and costs approximately $2 \%$ of the annual NHS budget [7]. People diagnosed with AHF typically have a poor prognosis, with a mortality rate of around $40 \%$ within a year of diagnosis [8], whereas for CHF patients, this rate is around 20\% [5, 9]. Overall, 5year survival rates for people with advanced HF are worse than for people with common forms of cancer like breast or prostate cancer [10].

The National Institute for Health and Care Excellence (NICE) guidelines [11] recommend the following actions as some of the key factors for improving quality of life, reduce hospitalisation frequency, and increase survival: early diagnosis, accurate assessment, providing prompt prognoses, and timely intervention [8, 12-14]. Current pharmacological and non-pharmacological interventions have been shown to increase the life expectancy of HF patients and reduce the number of related hospitalisations $[11,15]$. However, there has not been conclusive evidence supporting an improvement in hospitalisation rates in HFpEF [16]. Also, it has been demonstrated from clinical registry data that after each episode of acute HF, the prognosis of HF patients worsens, the risk of re-hospitalisation increases, and patients often do not receive optimised treatment (recommended care path, medication type, and dose for the individual's clinical characteristics) during or after each acute HF episode $[17,18]$. This is partly attributed to poor adherence to current guidelines [19] and a lack of widely accepted risk stratification models for $\mathrm{HF}[11,15,20]$.

\section{Prognostic factors and models}

Prognostic factors are clinical or biological patient characteristics that are related to certain disease outcomes. Biomarkers, which we define as biological factors measured in blood samples, may also serve as prognostic factors. In HF, the prognostic abilities of many biomarkers [21-25] have been investigated [22, 26]. Sometimes, multiple factors are combined into a prognostic model. As HF treatment decisions are generally based on a combination of symptoms and laboratory findings, by including the prognostic potential of multiple biomarkers, we may be able to better differentiate between individuals' needs and assist clinicians in offering maximum optimal HF treatment.

Prognostic models are commonly developed in individuals with a certain diagnosis (e.g. HF) to estimate their absolute risk of future disease outcomes [27]. They are mathematical expressions that combine multiple prognostic factors and can be used to guide treatment. A well-known example of a HF prognostic model is the Seattle Heart Failure Model (SHFM), which predicts 1-, 2 -, and 3-year survival using readily available clinical, therapy, and laboratory data [28]. Another example is the Meta-Analysis Global Group in Chronic Heart Failure Risk (MAGGIC) score which predicts 3-year survival based on similar factors to those in the SHFM [29]. 


\section{Potential health outcomes}

The use of prognostic models in disease management has several potential benefits [30]. For instance, model predictions can be used to inform important advanced care planning discussions with patients and their families, allowing treatment decisions to be individualised. Although some prognostic models focus on patient characteristics that are common or easy to obtain (e.g. age, gender, blood pressure levels), several studies have suggested that biomarkers such as adrenomedullin [21], high-sensitive cardiac troponin $\mathrm{T}$ (hs-cTnT) [22], cardiac troponin [23], soluble suppression of tumorigenicity-2 (sST2) [24], and galectin-3 [25] substantially improve their predictive performance. For this reason, prognostic models that require information on biomarkers are increasingly common in predicting clinical HF outcomes such as mortality, re-hospitalization, or advanced treatment (e.g. transplantation).

Although prognostic models are ideally developed using data from large prospective cohort studies, in practice, they are frequently derived using other available data sources such as randomised trials or databases with electronic health care records. As a result, published prognostic model studies may have limited generalisability or suffer from reduced data quality. Thus, before being introduced into clinical practice, it is essential that the predictive performance of these models is rigorously assessed in new samples (preferably from new settings) other that the one used for the model development. This requires assessment of the model's calibration, discrimination, and impact on external validation studies [28].

\section{Why this work is important}

Since the exploration of biomarkers became the norm first in the diagnosis and later in the prognosis of HF, there has been hundreds of prognostic models have been developed for HF. Ouwerkerk et al. in 2014 [31] summarised 117 models, while more recently Di Tanna et al. [32] identified a further 58 models published in a 5-year interval (2013 to 2018). Despite extensive work in the area, evidence on the validity and impact of these biomarker-based prognostic models on the clinical setting is lacking. Earlier systematic reviews [31, 33-35], while comprehensive in the inclusion of available models, were conducted before recent methodological advances in assessing [36], synthesising [37-39], and reporting $[40,41]$ prognostic models. More recent works while using up to date methodology, they have either restricted the models' publication date to a period of 5 years [32] or chose to present a discussion paper (rather than a systematic review) on selected models [42].

Concerns about bias was common to most previously published works, as was the reported inconsistent model performance in predicting mortality. In particular, existing HF models greatly differ in quality, target population, and measured outcomes. In addition, the predictive performance of these models is rarely assessed in new settings (especially calibration) [43]. Policy makers such as NICE and the European Society of Cardiology (ESC) have therefore been reluctant to recommend the use of any prognostic model in clinical guidelines [1]. However, it is possible that refraining from using any prognostic model to guide clinical practice can lead to suboptimal treatment decisions, and potentially even be worse than basing these decisions on an inaccurate prediction model. As a first step to resolve this conundrum, we propose to perform comprehensive reviews to identify prognostic models with biomarkers for clinical outcomes in adults with all types of HF and validations thereof, assess their methodological quality, and summarise their characteristics and predictive performance. The availability of novel prognostic methodology gives us the opportunity to re-evaluate the entire body of HF prognostic modelling literature, without restrictions on HF type, year of model publication, outcome assessed, or biomarkers explored.

\section{Methods}

The protocol is registered in PROSPERO (CRD42019086990) and follows the Preferred Reporting Items for Systematic Reviews and Meta-Analyses Protocols (PRISMA-P) 2015 statement [44] [see Additional file 3].

\section{Aims and objectives}

This project aims to (a) identify, describe, and appraise all developed prognostic models in HF involving at least one biomarker, as well as any subsequent validation studies and to (b) summarise available data in a metaanalysis to assess each models' predictive performance. To achieve these aims, we will conduct a number of systematic reviews to identify studies where a prognostic model has been developed and/or validated (either internally or externally), with or without any updating, according to the PICOTS items described in Table 1 . The outcomes of all systematic reviews planned, along with eligibility criteria for studies and population are also listed in Table 1.

We will summarise data only from prognostic models that predict either single or composite outcomes made up from two or more of the HF clinical outcomes stated in Table 1. Following standard systematic review metaanalysis will be attempted only in subsets of models with similar PICOTS and analysis methods. If meta-analysis is not possible results will be presented as a narrative. 
Table 1 PICOTS

Population Human adult patients aged 18 or older, diagnosed with any type of HF.

Intervention Multivariable models (i.e. models that contain two or more variables) for predicting any of the HF clinical outcomes listed below, or a (Model) combination of them, which considers, and possibly contains, prognostic factors, particularly biomarker concentrations, measured at baseline, on admission, or at discharge, or percentage change during hospitalization. The purpose of the model must be to yield absolute risk probabilities for individual patients. The biomarkers do not need to be part of the final model but considered as candidate predictors.

Outcomes a) Mortality (either all-cause mortality, sudden cardiac death, or death from progressive pump failure); b) HF-related hospitalisation; c) need for cardiac transplantation; d) mechanical assist device implantation, independent of other present co-morbidities; and e) major adverse cardiovascular events (MACE) such as non-fatal stroke, non-fatal myocardial infarction, and cardiovascular death. Any composite of these outcomes will also be considered

Timing No constraint will be imposed on the prediction horizon as this can vary according to the outcome predicted by each particular model. For instance, mortality could be predicted at 1, 2 or 3 years whereas re-hospitalisation could be predicted at 7 days, 1 months, or 6 months.

The timing of predictor measurements could be at diagnosis of HF, discharge after a HF-related hospitalisation, or start of study recruitment.

Setting Any setting relevant for the care of people with HF (e.g. primary care, hospital care, including emergency departments, cardiological departments, general medicine departments, intensive care units, or coronary care units).

\section{Inclusion and exclusion criteria}

Table 2 lists the inclusion and exclusion criteria, separately for the type of studies and the target population.

\section{Information sources}

We will search the following databases from 1990 onwards, as the biomarkers' assays were first conducted in the 1990s, with no language restriction to reduce potential bias: MEDLINE (OvidSP); EMBASE (OvidSP); Science Citation Index \& Conference Proceedings Citation Index-Web of Science Core Collection (Wok); and Database of prognostic studies maintained by the
Cochrane Prognosis Methods Group (PMG). We will screen the reference lists of the included studies, relevant review articles, and practice guidelines. Authors of relevant studies, study groups, experts and investigators known to be active in the field will be contacted for unpublished material or further information on ongoing studies.

\section{Search strategy}

We will aim for broad literature searches by targeting studies that focus on investigating prognosis in HF patients, and hence will combine published search filters for a sensitive search strategy [45]. Additional file 1

Table 2 Eligibility criteria

\section{Criteria Type of studies}

Inclusion We will include only primary clinical studies in HF with clinical models and/or outcomes that present:

- Prognostic model development, adjustment or updating with or without external validation. The model's discrimination and/or calibration must have been reported.

- External model validation. The model's discrimination and/or calibration must have been reported. The source of data could be medical records, existing RCT data, or large clinical databases.

Exclusion - Studies using exclusively assay analyses.

- Studies published only as abstracts or clinical trials reporting no prognostic modelling on HF patients.

- Studies developing models with the sole intention of evaluating the independent or adjusted association of a factor (even if this is a biomarker) with the outcome and not to predict individual probabilities.

- Studies that explore the prognostic effect of treatment (eg medication regimes, device implantation, etc.)

- Systematic reviews, unless authors use a review to form a data repository for developing a prognostic model.. Their citation list will be explored for further inclusion of primary studies potentially missed by our sensitive search.

- Literature reviews.

- Case studies.

- Diagnostic studies.

- Studies focusing on economic evaluations of HF care.

\section{Target population}

Adult patients aged 18 or older, diagnosed with any type of HF (i.e. ischaemic, non-ischaemic, chronic, acute, or decompensated). Patients with both reduced and preserved ejection fraction HF are eligible for inclusion. Those patients may or may not have already received optimum medical therapy (OMT), including medications and implantable devices (e.g. implantable cardioverter defibrillator (ICD) and cardiac resynchronization therapy (CRT) devices).

- Patients who are recipients or already registered candidates for transplantation, left (LVAD), or biventricular (BiVAD) assist devices as their HF status will be significantly altered by this intervention. - Patients with advanced/end stage HF (e.g. NYHA IV) and those receiving end of life or palliative care, or who also suffer from HF as a comorbidity will not be considered because of their already established poor prognosis.

- Patients with HF due to congenital conditions, and secondary to reversible causes (such as valvular disease, pregnancy and peripartum, infection, major surgery, pre-revascularisation, intensive care conditions).

- Patients with concomitant disease which predominantly affects prognosis, such as cancer and neurological disorders. 
presents the search strategy. Searches will be carried out by a health information specialist (NR).

\section{Study records}

\section{Data management}

Screening will be performed using Covidence [46] and selected articles (including their portable document format (PDF) files) will be managed using EndNote X8.

\section{Selection process}

Pairs of authors will independently screen titles and abstracts for eligibility, followed by full text assessment. In the case of disagreement, a third reviewer will be consulted [47]. We will document the total numbers of retrieved references and the numbers of included and excluded studies in a flow chart, as recommended in the Preferred Reporting Items for Systematic Reviews and Meta-Analyses (PRISMA) statement [48].

\section{Data collection process}

In pairs, we will independently extract data according to a piloted form that will combine adapted versions of the Critical Appraisal and Data Extraction for Systematic Reviews of Prediction Modelling studies (CHARMS) checklist [38] to assess the methodological quality conduct of the included prognostic models and the Prediction Model Risk of Bias Assessment Tool (PROBAST) [36].

\section{Data items}

We will collect the following data about the selected studies and models:

- General information-author, title, source, publication date

- Source of data-for example, existing cohort, registry data

- Participants' information-eligibility and recruitment method, study dates, treatments received, ethnicity, age and sex distributions

- Outcomes to be predicted-definition, blinding and time of measurement

- Candidate predictors-number, biomarkers included, and variables in the final model or model being validated. A list of potential biomarkers [see Additional file 2] that models might have considered will be included in the extraction form, with an option to record any additional HF-related biomarker encountered

- Information on missing data

- Model development-total sample size, total number of events, model name (if any), modelling method, assumptions assessment, predictors selection prior and during modelling, use of shrinkage techniques, testing for interactions, handling of continuous predictors

- Reporting of model-whether reported the final and other multivariable models including predictor weights, intercept, baseline survival (when appropriate), model performance measures (with standard errors or confidence intervals), and any alternative presentation of the final model

- Model validation-total sample size, total number of events, validation procedure (e.g. apparent, split-sample, other type of internal validation, external validation)

- Internal validation-whether it was an apparent validation (i.e. without applying resampling techniques or hold-outs) or proper internal validation, i.e. using resampling methods (e.g., bootstrap or cross-validation) for building the model and not only for the final model. We will report if values have been adjusted for optimism.

- External validation-target population, setting, data collection procedures. In cases of disappointing performance in external validation samples, we will report whether the model was updated in response, e.g. intercept recalibrated, predictor effects adjusted, or new predictors added. In cases of external validation, we will compare the list and distribution of predictors (that is, the mean and standard deviation, as well as the presence of missing data and/or missing predictors) for development and validation datasets, considering those of the development study as the reference.

- Model performance measures-calibration, discrimination, and overall performance measures. We will extract the corresponding estimates together with their standard error, 95\% confidence interval, and (if applicable) $p$ values, when reported and as appropriate. For calibration-the model's ability to generate predicted probabilities similar to the observed probabilities-we will describe whether calibration plots, calibration slope, calibration intercept, Hosmer-Lemeshow goodness of fit test (for logistic models), and/or observed/expected outcomes ratio (O/E ratio) are reported. For discrimination-the model's ability to correctly classify patients with and without the outcome of interest-we will report whether the area under the receiver operating characteristic (ROC) curve (AUC), concordance (c-index) statistic, D-statistic, and/or the log-rank test are presented. We will also report if other performance measures are presented, including $R^{2}$ and the Brier score.

\section{Missing data}

We will contact authors of individual studies for additional information, if required, particularly when there 
are missing performance measures and their variation estimates (i.e. standard deviation, and 95\% confidence intervals). If such information does not become available, we will collect the following information instead, according to Debray et al.: [37]

- If no calibration measures are reported, we will extract information on: the mean predictor values (usually presented together with the sample characteristics); predicted number of events for the overall sample and/or predicted outcome probability and observed outcome probability (to be estimated from Kaplan-Meier curves in the presence of censoring); when available, observed and predicted outcomes across risk strata and/or observed and predicted outcome probabilities across risk strata.

- If no discrimination values are reported, we will extract information on: the distribution of the linear predictor (LP, i.e. linear combination of the model predictors in the study sample weighted by the regression coefficients of the model in the development study), i.e. overall variance of the LP; mean and standard deviation of the LP in individuals with the outcome; and mean and standard deviation of the LP in individuals without the outcome.

This information will allow us to estimate $\ln (\mathrm{O} / \mathrm{E})$ and its variance and the logit(c) and its variance, quantities required for the meta-analysis of calibration and discrimination, respectively. These estimates will be obtained using the methods implemented in the $\mathrm{R}$ package metamisc [49]. If three or more studies are available and are clinically homogenous (e.g. similar prognostic factors, outcomes, prediction horizons, study conduct, purpose, quality), the same package will be used to meta-analyse model performance.

\section{Assessing risk of bias}

The risk of bias in individual studies will be assessed using the Prediction Model Risk of Bias Assessment Tool (PROBAST) [36], which was developed to evaluate the extent to which shortcomings in the study design, conduct and analysis yield over- or under-estimated model predictive performance values. PROBAST also evaluates the applicability or extent to which the prognostic study assessed matches the systematic review research question in terms of population, predictors, and outcomes. PROBAST consists of 20 signalling questions grouped in four domains: participant selection; predictors; outcome; and analysis. The individual items of this tool will be embedded in the relevant sections of this review's data-extraction form. An overall judgement will be made, reporting a 'low', 'high' or 'unclear' risk of bias and 'low', 'high', or 'unclear' concerns regarding applicability according to the tool guidelines.

\section{Publication bias}

Unlike randomised control trial studies, prognostic modelling studies are typically not prospectively registered and usually no protocol is published [50]. Although difficult to estimate from reported data, we will evaluate and discuss the potential presence of publication bias.

\section{Data analysis and synthesis}

For each HF prognostic model identified by our search strategy, we will tabulate the following information: participant population (specifying type of HF, setting and total sample size), model (name or brief description if no name available, type of statistical model, number of prognostic factors, biomarker(s) investigated, discrimination, calibration, internal validation method and presentation format of the model), and outcome (type, definition, prediction horizon and number of events).

For prognostic models that have been externally validated, an additional tabular display will be used to show: validation study identifier; participant population (specifying type of HF); setting; whether all prognostic factors in the original model were available and similarly measured in the external validation population; whether the original mathematical expression was used to estimate outcome probabilities; number of events/sample size; discrimination; calibration; any updates to the model.

This project plan consists of a number of systematic reviews. Hence, we will not pool all findings in one report but rather, we will focus on a subset of studies (models) where a summary and/or meta-analysis are feasible and informative. The hierarchy of decisions will start form HF types, go down to summarising derivation models grouped by clinical outcome reported, and finally carry out meta-analysis of performance estimates (extracted from external validation studies) of one model and one outcome (single or composite as per Table 1) at a time.

More specifically if sufficient data are available and if the corresponding studies have a fair degree of similarities in terms of their PICOTS, we will meta-analyse the predictive performance estimates of each model, provided that their risk of bias is negligible, using random effects models with weights given by the within-study error variance, to account for the expected amount of between-study heterogeneity. To obtain accurate summary estimates and to avoid excluding studies with poor reporting of performance measures, we will use multivariate meta-analysis [37]. If a particular model has been validated in three or more occasions, we will pool the results by applying meta-analyses and meta-regression. 
Meta-analyses will be performed using the $R$ packages metamisc, and metafor (for meta-regression) [49].

As a sensitive search strategy will be used, we expect to observe a large amount of clinical as well as statistical and design heterogeneity amongst included studies. For each type of HF, we will explore the impact of the following design features known to affect the predictive performance of prognostic models for studies reporting models that contain similar predictors:

- Participants characteristics, including study dates to cover for improvements in biomarker measurement techniques, and study setting (e.g. primary or secondary care)

- Outcome definition, method and measurement time

- Number of candidate predictors, predictor selection methods, and handling of predictors

- Sample size and number of events

- Handling of missing data

- Type of reported predictive performance measures

- Differences between development and external validation populations

Overall between-study heterogeneity, particularly for performance measures of calibration and discrimination, will be assessed using the $I^{2}$ statistic. Because this measure can be misleading, we will complement the assessment estimating Kendall's tau and approximate 95\% prediction intervals (which provide a range for the potential performance in a new validation study) will be calculated to further interpret the relevance of any between-study heterogeneity [50].

If ten or more studies are available, we will perform meta-regression analyses, where feasible, for biomarker(s); prediction horizon; setting; co-morbidities; studies assessing the performance of original models; studies assessing the performance of updated models (recalibrated or adjusted); studies assessing particular models.

Potential methodological influences will be explored using sensitivity analysis by temporarily removing from the analysis studies with high risk of bias for at least one domain of PROBAST. If study quality is low or if the between-study heterogeneity is high, we will report results as a narrative.

\section{Summary of findings}

Currently, we are not able to assess the quality of the evidence using the GRADE (Grading of Recommendations, Assessment, Development and Evaluations) process, as GRADE guidance for prognostic models has not been developed yet. Instead, we will present in our summary of findings the biomarkers included in each model, the original and updated models, their predictive performance (apparent, internal, and external, if reported), population characteristics, most common predictor factors, and the clinical outcomes considered in this review that are listed in Table 1.

\section{Discussion}

This project will consist of a number of systematic reviews that will allow us to assess the characteristics of prognostic models for HF which consider and/or include essential biomarkers, appraise their methodological conduct, and that of subsequent studies assessing the models' predictive performance in populations other than the one used for the models' development (referred to as external validation).

We envisage a very high yield of titles from the searches, from which only a small percentage will be eligible for inclusion. This is because the current recommended prognostic filters [33] include very broad criteria, hence the high yield. From a scoping search, we found that approximately $6 \%$ of the titles of an original search would be eligible for inclusion.

Additionally, it is anticipated that selecting the eligible papers may require training the not-statistically minded team members in prognostic modelling matters.

If sufficient data are available from the eligible studies, we will meta-analyse the models' predictive performance. This evidence will guide future HF prognostic model design and contribute to improved HF clinical management.

Any important future protocol amendments as a result of insight acquired during the project development stages, will be documented in detail in a separate section titled 'Differences from original protocol' and justification for all changes will be offered.

\section{Supplementary information}

Supplementary information accompanies this paper at https://doi.org/10. 1186/s41512-020-00081-4.

Additional file 1. Heart failure prognostic biomarker models searches This file contains the search strategies used to identify relevant studies in MEDLINE, EMBASE, and WoK

Additional file 2. List of potential biomarkers. This file contains a nonexhaustive list of possible HF-related biomarkers a prognostic HF model could include at the start of the model development process or retained in the final stage

Additional file 3. Preferred reporting items for systematic review and meta-analysis protocols (PRISMA-P) 2015

\section{Abbreviations}

AHF: Acute heart failure; AUC: Area under the receiver operating characteristic (ROC) curve; BiVAD: Biventricular assist device; BNP: B-type natriuretic peptide; CHARMS: Critical Appraisal and Data Extraction for Systematic Reviews of Prediction Modelling studies; CHF: Chronic heart failure; CRT: Cardiac resynchronization therapy device; EMBASE: Excerpta Medica database; ECS: European Society of Cardiology; HF: Heart failure; hsCTnT: High-sensitive cardiac troponin T; ICD: Implantable cardioverterdefibrillator device; LP: Linear predictor, i.e: linear combination of the model 
predictors in the study sample weighted by the regression coefficients of the model in the development study; LVAD: Left ventricular assist device." MACE: Major adverse cardiovascular events; MAGGIC: Meta-Analysis Global Group in Chronic Heart Failure; NHS: National Health Service; NICE: National Institute for Health and Care Excellence; NT-proBNP: N-terminal prohormone B-type natriuretic peptide; O/E ratio: Observed/expected outcomes ratio; OMT: Optimum medical therapy; PMG: Cochrane Prognosis Methods Group; PRISMA: Preferred Reporting Items for Systematic Reviews and Meta-Analyses statement; PROBAST: Prediction Model Risk of Bias Assessment Tool; PROSPERO: International Prospective Register of Systematic Reviews; SHFM: Seattle Heart Failure Model; soluble ST2 or SST2: Soluble suppression of tumorigenicity-2; UK: United Kingdom; US: United States; WoK: Web of Science Core Collection

\section{Acknowledgements}

We are grateful to the late Prof Doug Altman for his valuable advice at the conception and protocol development stages of this project.

\section{Authors' contributions}

NPEK and MT conceived the study, and along with MVM drafted the protocol. KST, RP, TD, MVM, KM, and MT provided input on methodological issues; and NK, FDRH, JP, and CT on clinical issues. The search strategy was developed and applied by NR. All authors provided advice and input regarding the protocol, and have contributed, read, and approved the final manuscript. MT is the guarantor of the article.

\section{Funding}

This work is embedded in the Systematic Reviews on the Prognostic Role of Biomarkers in Heart Failure (proBHF) project funded by the British Heart Foundation (grant no. PG/17/49/33099). MVM, MT, KT, and NPEK are supported or partly supported by this grant, while CT, FDRH, GSC, KGMM, JP, and RP are named co-applicants and collaborators in the same grant. MT is the PI for this project grant.

$\mathrm{CT}$ is a National Institute for Health Research (NIHR) Academic Clinical Lecturer. CT and FDRH are co-leads for the chronic disease theme of the Oxford NIHR Medtech In vitro diagnostics Co-Operative (MIC).

FDRH acknowledges his part-funding from the National Institute for Health Research (NIHR) School for Primary Care Research, the NIHR Collaboration for Leadership in Health Research and Care (CLARHC) Oxford, the NIHR Oxford Biomedical Research Centre (BRC, Oxford University Hospitals Trust), and the NIHR Oxford Medtech and In-Vitro Diagnostics Co-operative (MIC).

BS is supported by an Advanced Postdoc Mobility grant from the Swiss National Science Foundation (P300PB_177933).

$\mathrm{NJ}$ is supported by a Wellcome Trust Doctoral Research Fellowship [grant number 203921/Z/16/Z]

The views expressed are those of the authors and not necessarily those of the NHS, the NIHR, or the Department of Health and Social Care.

The funding bodies had no role in study design, data collection, analysis, interpretation, or in the writing of the manuscript.

\section{Availability of data and materials}

Not applicable. This is only a protocol—no data were generated or used.

\section{Ethics approval and consent to participate}

Not applicable.

\section{Consent for publication}

Not applicable.

\section{Competing interests}

CT declares speaker fees from Novartis and Vifor outside the submitted work and non-financial support from Roche outside the submitted work. All other authors declare that they have no competing interests.

\section{Author details}

'Nuffield Department of Primary Care Health Sciences, Radcliffe Observatory Quarter, University of Oxford, Woodstock Road, Oxford OX2 6GG, UK. ${ }^{2}$ Centre for Statistics in Medicine, Nuffield Department of Orthopaedics,

Rheumatology and Musculoskeletal Sciences, Botnar Research Centre, University of Oxford, Windmill Road, Oxford OX3 7LD, UK. ${ }^{3}$ Julius Center for Health Sciences and Primary Care, University Medical Center (UMC), Utrecht
University, Heidelberglaan 100, 3584, CX, Utrecht, The Netherlands. ${ }^{4}$ Basel Institute for Clinical Epidemiology and Biostatistics, Department of Clinical Research, University Hospital Basel, University of Basel, Basel, Switzerland. ${ }^{5}$ National Institute for Health Research Oxford Biomedical Research Centre, John Radcliffe Hospital, Oxford, UK. ${ }^{6}$ Department of Food Science and Nutrition, Agricultural University of Athens, lera Odos, 75 Athens, Greece. ${ }^{7}$ School of Mathematical Sciences, Queen Mary University of London, E1 4NS, London, UK. ${ }^{8}$ Second Department of Cardiology, Attikon University Hospital, National and Kapodistrian University of Athens, Athens, Greece. ${ }^{9}$ Bodleian Health Care Libraries, University of Oxford, Oxford, UK.

Received: 15 January 2020 Accepted: 23 July 2020

Published online: 26 August 2020

\section{References}

1. Ponikowski P, Anker SD, AlHabib KF, Cowie MR, Force TL, Hu S, et al. Heart failure: preventing disease and death worldwide. ESC heart failure. 2014;1(1):4-25.

2. Mehra MR, Maisel A. B-type natriuretic peptide in heart failure: diagnostic, prognostic, and therapeutic use. Crit Pathw Cardiol. 2005;4(1):10-20.

3. Rosenzweig A, Seidman CE. Atrial natriuretic factor and related peptide hormones. Annu Rev Biochem. 1991;60:229-55.

4. Mozaffarian D, Benjamin EJ, Go AS, Arnett DK, Blaha MJ, Cushman M, et al. Heart disease and stroke statistics-2016 update a report from the American Heart Association. Circulation. 2016;133(4):e38-48.

5. Jones NR, Roalfe AK, Adoki I, Hobbs FR, Taylor CJ. Survival of patients with chronic heart failure in the community: a systematic review and metaanalysis. European Journal of Heart Failure. 2019.

6. Ross JS, Chen J, Lin Z, Bueno H, Curtis JP, Keenan PS, et al. Recent national trends in readmission rates after heart failure hospitalization. Circ Heart Fail. 2010;3(1):97-103.

7. NICE. Chronic heart failure-Costing report-Implementing NICE guidance National Institute for Health and Care Excellence 2010. Full guideline in: https://www.nice.org.uk/guidance/cg108/evidence/fullguideline-136060525; 2010.

8. Maisel AS, Peacock WF, McMullin N, Jessie R, Fonarow GC, Wynne J, et al. Timing of immunoreactive B-type natriuretic peptide levels and treatment delay in acute decompensated heart failure: an ADHERE (Acute Decompensated Heart Failure National Registry) analysis. J Am Coll Cardiol. 2008;52(7):534-40.

9. Taylor CJ, Ryan R, Nichols L, Gale N, Hobbs FR, Marshall T. Survival following a diagnosis of heart failure in primary care. Fam Pract. 2017;34(2):161-8.

10. Askoxylakis V, Thieke C, Pleger ST, Most P, Tanner J, Lindel K, et al. Long term survival of cancer patients compared to heart failure and stroke: a systematic review. BMC cancer. 2010;10:105.

11. NICE. National Clinical Guideline Collaborating Centre. Chronic heart failure in adults: management. National Institute for Health and Care Excellence 2010. Full guideline in: https://www.nice.org.uk/guidance/cg108/evidence/ full-guideline-136060525; 2010.

12. Maisel A, Mueller C, Nowak RM, Peacock WF, Ponikowski P, Mockel M, et al. Midregion prohormone adrenomedullin and prognosis in patients presenting with acute dyspnea: results from the BACH (Biomarkers in Acute Heart Failure) trial. J Am Coll Cardiol. 2011;58(10):1057-67.

13. Maisel A, Mueller C, Nowak R, Peacock WF, Landsberg JW, Ponikowski P, et al. Mid-region pro-hormone markers for diagnosis and prognosis in acute dyspnea: results from the BACH (Biomarkers in Acute Heart Failure) trial. J Am Coll Cardiol. 2010;55(19):2062-76.

14. O'Donoghue ML, Morrow DA, Cannon CP, Jarolim P, Desai NR, Sherwood $M W$, et al. Multimarker risk stratification in patients with acute myocardial infarction. J Am Heart Assoc. 2016;5(5).

15. NICE. National Clinical Guideline Collaborating Centre. Acute heart failure: diagnosis and management. National Institute for Health and Care Excellence 2014. Full Guideline in: https://www.nice.org.uk/guidance/cg187/ evidence/full-guideline-193260781; 2014.

16. Dunlay SM, Roger VL, Redfield MM. Epidemiology of heart failure with preserved ejection fraction. Nature Reviews Cardiology. 2017;14(10):591.

17. Kitamura K. Adrenomedullin and related peptides. Nihon Yakurigaku Zasshi. 1998;112(3):137-46.

18. Nishikimi T, Saito Y, Kitamura K, Ishimitsu T, Eto T, Kangawa K, et al. Increased plasma levels of adrenomedullin in patients with heart failure. J Am Coll Cardiol. 1995;26(6):1424-31. 
19. Ponikowski P, Voors AA, Anker SD, Bueno H, Cleland JG, Coats AJ, et al. ESC Guidelines for the diagnosis and treatment of acute and chronic heart failure: The Task Force for the diagnosis and treatment of acute and chronic heart failure of the European Society of Cardiology (ESC). Developed with the special contribution of the Heart Failure Association (HFA) of the ESC. Eur J Heart Fail. 2016, 2016.

20. NICE. Chronic heart failure in adults: diagnosis and management. National Institute for Health and Care Excellence 2018. Full guideline in: https://www. nice.org.uk/guidance/ng106/resources/chronic-heart-failure-in-adultsdiagnosis-and-management-pdf-66141541311685; 2018.

21. Funke-Kaiser A, Mann K, Colquhoun D, Zeller T, Hunt D, Simes J, et al. Midregional proadrenomedullin and its change predicts recurrent major coronary events and heart failure in stable coronary heart disease patients: the LIPID study. Int J Cardiol. 2014;172(2):411-8.

22. Demissei BG, Cotter G, Prescott MF, Felker GM, Filippatos G, Greenberg BH, et al. A multimarker multi-time point-based risk stratification strategy in acute heart failure: results from the RELAX-AHF trial. Eur J Heart Fail. 2017.

23. Jungbauer CG, Riedlinger J, Buchner S, Birner C, Resch M, Lubnow M, et al. Highsensitive troponin $\mathrm{T}$ in chronic heart failure correlates with severity of symptoms, left ventricular dysfunction and prognosis independently from N-terminal pro-btype natriuretic peptide. Clin Chem Lab Med. 2011;49(11):1899-906.

24. Gandhi PU, Testani JM, Ahmad T. The current and potential clinical relevance of heart failure biomarkers. Curr Heart Fail Rep. 2015:12(5):318-27.

25. Filipe MD, Meijers WC. Rogier van der Velde A, de Boer RA. Galectin-3 and heart failure: prognosis, prediction \& clinical utility. Clin Chim Acta. 2015:443:48-56.

26. Jackson CE, Haig C, Welsh P, Dalzell JR, Tsorlalis IK, Mc Connachie A, et al. The incremental prognostic and clinical value of multiple novel biomarkers in heart failure. Eur J Heart Fail. 2016.

27. Steyerberg EW, Moons KG, van der Windt DA, Hayden JA, Perel P, Schroter $S$, et al. Prognosis Research Strategy (PROGRESS) 3: prognostic model research. PLoS Med. 2013;10(2):e1001381.

28. Levy W, Mozaffarian D, Linker D, Sutradhar S, Anker S, Cropp A. The Seattle Heart Failure Model: prediction of survival in heart failure. Circulation [Internet]. 2006 [citado: 12/12/2016]; 113:[aprox. 6 p.].

29. Pocock SJ, Ariti CA, McMurray JJ, Maggioni A, Køber L, Squire IB, et al. Predicting survival in heart failure: a risk score based on 39372 patients from 30 studies. European heart journal. 2012;34(19):1404-13.

30. Hemingway H, Croft P, Perel P, Hayden JA, Abrams K, Timmis A, et al. Prognosis research strategy (PROGRESS) 1: a framework for researching clinical outcomes. Bmj. 2013;346:e5595.

31. Ouwerkerk W, Voors AA, Zwinderman AH. Factors influencing the predictive power of models for predicting mortality and/or heart failure hospitalization in patients with heart failure. JACC Heart Fail. 2014;2(5):429-36.

32. Di Tanna GL, Wirtz H, Burrows KL, Globe G. Evaluating risk prediction models for adults with heart failure: A systematic literature review. PLoS One. 2020;15(1):e0224135.

33. Rahimi K, Bennett D, Conrad N, Williams TM, Basu J, Dwight J, et al. Risk prediction in patients with heart failure: a systematic review and analysis. JACC Heart Fail. 2014;2(5):440-6.

34. Alba AC, Agoritsas T, Jankowski M, Courvoisier D, Walter SD, Guyatt GH, et al. Risk prediction models for mortality in ambulatory patients with heart failure: a systematic review. Circ Heart Fail. 2013;6(5):881-9.

35. Ross JS, Mulvey GK, Stauffer B, Patlolla V, Bernheim SM, Keenan PS, et al. Statistical models and patient predictors of readmission for heart failure: a systematic review. Arch Intern Med. 2008;168(13):1371-86.

36. Wolff RF, Moons KGM, Riley RD, Whiting PF, Westwood M, Collins GS, et al. PROBAST: A tool to assess the risk of bias and applicability of prediction model studies. Annals of Internal Medicine. 2019;170(1):51-8.

37. Debray TP, Damen JA, Snell KI, Ensor J, Hooft L, Reitsma JB, et al. A guide to systematic review and meta-analysis of prediction model performance. bmj. 2017;356:16460.

38. Moons KG, de Groot JA, Bouwmeester W, Vergouwe Y, Mallett S, Altman $D G$, et al. Critical appraisal and data extraction for systematic reviews of prediction modelling studies: the CHARMS checklist. PLOS medicine. 2014; 11(10):e1001744

39. Debray TP, Koffijberg H, Nieboer D, Vergouwe Y, Steyerberg EW, Moons KG. Meta-analysis and aggregation of multiple published prediction models. Stat Med. 2014;33(14):2341-62.

40. Collins GS, Reitsma JB, Altman DG, Moons KGM. Transparent Reporting of a multivariable prediction model for Individual Prognosis Or Diagnosis
(TRIPOD): The TRIPOD StatementThe TRIPOD Statement. Annals of Internal Medicine. 2015;162(1):55-63.

41. Moons KGM, Altman DG, Reitsma JB, loannidis JPA, Macaskill P, Steyerberg EW, et al. Transparent Reporting of a multivariable prediction model for Individual Prognosis Or Diagnosis (TRIPOD): Explanation and ElaborationThe TRIPOD Statement: Explanation and Elaboration. Annals of Internal Medicine. 2015;162(1):W1-W73.

42. Doumouras BS, Lee DS, Levy WC, Alba AC. An appraisal of biomarker-based risk-scoring models in chronic heart failure: which one is best? Current Heart Failure Reports. 2018;15(1):24-36.

43. Collins GS, de Groot JA, Dutton S, Omar O, Shanyinde M, Tajar A, et al. External validation of multivariable prediction models: a systematic review of methodological conduct and reporting. BMC Med Res Methodol. 2014;14:40

44. Moher D, Shamseer L, Clarke M, Ghersi D, Liberati A, Petticrew M, et al. Preferred reporting items for systematic review and meta-analysis protocols (PRISMA-P) 2015 statement. Systematic reviews. 2015:4(1):1.

45. Geersing G-J, Bouwmeester W, Zuithoff P, Spijker R, Leeflang M, Moons K. Search filters for finding prognostic and diagnostic prediction studies in Medline to enhance systematic reviews. PLoS One. 2012;7(2):e32844.

46. Covidence systematic review software VHI, Melbourne, Australia. [Available from: www.covidence.org.

47. Higgins J, Deeks JJ. Selecting studies and collecting data. Cochrane Handbook for Systematic Reviews of Interventions: Cochrane Book Series. 2008:151-85.

48. Moher D, Liberati A, Tetzlaff J, Altman DG, Group P. Preferred reporting items for systematic reviews and meta-analyses: the PRISMA statement. PLoS medicine. 2009;6(7):e1000097.

49. Debray T. Package 'metamisc' 2018 [Available from: https://CRAN.R-project. org/package $=$ metamisc .

50. Harrell FE. Ordinal logistic regression. Regression modeling strategies: Springer; 2015. p. 311-325.

\section{Publisher's Note}

Springer Nature remains neutral with regard to jurisdictional claims in published maps and institutional affiliations.

Ready to submit your research? Choose BMC and benefit from:

- fast, convenient online submission

- thorough peer review by experienced researchers in your field

- rapid publication on acceptance

- support for research data, including large and complex data types

- gold Open Access which fosters wider collaboration and increased citations

- maximum visibility for your research: over $100 \mathrm{M}$ website views per year

At $\mathrm{BMC}$, research is always in progress.

Learn more biomedcentral.com/submissions 